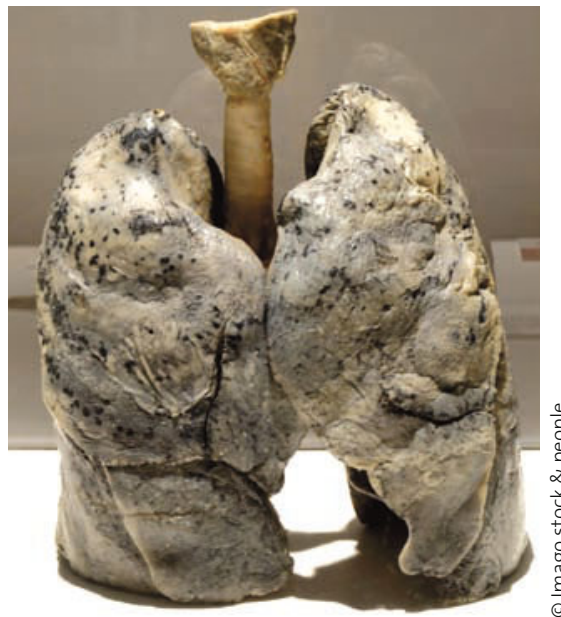

Den Schaden an der Lunge anschaulich machen.

- Nach dem 25. Lebensjahr nimmt die Lungenleistung kontinuierlich ab. Bei Rauchern vollzieht sich dieser Abfall der Lungenfunktion überproportional

Motivation zur Nikotinentwöhnung Rechnen Sie dem Patienten vor, wie alt seine Lunge wirklich ist!

schnell. Das biologische Alter der Lunge eines Rauchers im mittleren Lebensalter kann durchaus dem biologischen Lungenalter eines hochbetagten Nichtrauchers entsprechen. Rauchern diesen Zusammenhang klar zu machen, wirkt oft sehr motivierend, ernsthaft an einem Programm zur Nikotinentwöhnung teilzunehmen.

Die Motivation ist umso besser, je konkreter man dem Raucher verdeutlichen kann, wie vorgealtert seine Lunge schon ist. Sehr gut gelingt dies anhand einer Kurve, die der britische Epidemiologe Sir Richard Peto erarbeitet hat. Sie zeigt den Abfall der Lungenfunkti- on bei Rauchern und bei Nichtrauchern sowie bei Exrauchern. Mit einer mathematischen Formel lässt sich in Abhängigkeit vom gemessenen $\mathrm{FEV}_{1}$-Wert und dem Geschlecht rasch das konkrete biologische Alter der Lunge errechnen. Die Formel gibt es als Excel-Datei unter www.lungenzentrum-ulm.de (unter Praxisservice) oder als I-Phone App „lung-age“. Weist beispielsweise ein 55jähriger Raucher einen $\mathrm{FEV}_{1}$-Wert von $40 \%$ auf, entspricht das einem Lungenalter von rund 90 Jahren beim Nichtraucher.

DR. MICHAEL BARCZOK, ULM =

\section{OBSTRUKTIVE ATEMWEGSERKRANKUNGEN}

Fünf Phänotypen, die Sie kennen sollten

Obstruktive Atemwegserkrankungen lassen sich nach ihrem Phänotyp unterscheiden. Diese Einteilung deckt sich nach neuen Daten nicht mit der klassischen Einteilung in Asthma, chronische Bronchitis und Emphysem deckt. Eine große Studie aus Neuseeland konnte fünf verschiedene Phänotypen identifizieren:

(1) Emphysem ohne Asthma oder chronische Bronchitis mit schwerer Atemwegsobstruktion, meist verbunden mit starkem Rauchen

2 Milde messbare Atemwegsobstruktion

(3) Atopisches Asthma, chronische Bronchitis und Emphysem mit schwerer reversibler Atemwegsobstruktion, PeakflowVariabilität, assoziiert mit Rauchen

(4) Atopisches Asthma mit Ekzem und allergischer Rhinitis; eosinophile Atemwegsentzündung, erhöhte NO-Konzentration im Exhalat

(5) Atemwegsobstruktion, chronischer Auswurf, Ekzem, Rhinitis mit Eosinophilie im Blut und NO-Anstieg im Exhalat

Die derzeitigen Diagnosekriterien und Therapieempfehlungen für Asthma und COPD werden einem erheblichen Teil dieser Patienten nicht hinreichend gerecht. Ein Beispiel aus der Therapie: Steroide sind eingeschränkt wirksam bei Astmapatienten, die rauchen. Die Response auf Steroide kann bei diesen Patienten durch niedrig dosiertes Theophyllin gesteigert werden.

\section{LUNGENFUNKTION ERHALTEN}

\section{Am besten vor dem 30. Geburtstag aufhören zu rauchen!}

Auch ein anderes pneumologisches Dogma gerät derzeit ins Wanken. Es lautet: Der durch Rauchen langfristig verursachte Abfall der Lungenfunktion kann abgeflacht werden, wenn man mit dem Rauchen aufhört - egal zu welchem Zeitpunkt der Raucherkarriere dies geschieht.

Eine neue Untersuchung einer großen Kohorte von Nachkommen der Framingham-Population (R. Kohansal et al., AJRCCM 2009;180:3-10), in der über einen langen Beobachtungszeitraum standardisierte Spirometrien durchgeführt wurden, spricht dafür, dass dies nicht korrekt ist. Vielmehr lassen die Daten darauf schließen, dass der Verlust von Lungenfunktion nur dann nennenswert aufgehalten werden kann, wenn es gelingt, vor einem Alter von 30 Jahren mit dem Rauchen aufzuhören.

$A B=$

- 6. Kongress der Deutschen Gesellschaft für Innere Medizin (DGIM), Wiesbaden, 10.-14. April 2010 